Supporting Information Available

\title{
Synthesis of Tellurium Fusiform Nano-Architectures by Controlled Living Nanowire Modification
}

Yunpeng Zuo ${ }^{\dagger a}$, Tingting Li ${ }^{\dagger a}$, Dewei Rao ${ }^{\dagger b}$, Xiaomin Lei ${ }^{a}$, Qin Li ${ }^{a}$, Guilin Zhu ${ }^{a}$, Ruifeng Lu ${ }^{c}$ and Heyou Han $^{* a}$

${ }^{\dagger}$ These authors contributed equally to this work.

${ }^{a}$ State Key Laboratory of Agricultural Microbiology, College of Science, Huazhong Agricultural University, Wuhan 430070, P R China. E-mail: hyhan@mail.hzau.edu.cn

${ }^{b}$ Institute for Advanced Materials, Jiangsu University, Zhenjiang 212013, P R China.

${ }^{c}$ Department of Applied Physics, Nanjing University of Science and Technology, Nanjing 210094, P R China.

"To whom correspondence should be addressed. Tel: +86-27-87282043; fax: $+86-27-87282043$.

E-mail: hyhan@mail.hzau.edu.cn 


\section{Experimental Details}

Chemicals: Hexadecyltrimethylammonium bromide (CTAB, 99\%), sodium dodecyl sulfate (SDS, 99\%) were purchased from Sigma-Aldrich. Tellurium dioxide powder $\left(\mathrm{TeO}_{2}, 99.99 \%\right)$ was purchased from Aladdin Chemistry Co., Ltd. Hydrazine monohydrate (85\%, AR) was supplied by Sinopharm Chemical Reagent Co., Ltd. Unless special instructions, other chemicals were of analytical reagent. Ultrapure water for the whole experiment process was with a conductivity of $18.25 \mathrm{M} \Omega \mathrm{cm}$.

Apparatus: Transmission electron microscopy (TEM) and high-resolution transmission electron microscopy (HRTEM) measurements were made on a JEM-2010FEF high-resolution transmission electron microscopy at an accelerating voltage of $200 \mathrm{kV}$. The compositions of Te nanomaterials were determined by ICP AES (ELAN 6000, Perkin-Elmer). The X-ray diffraction (XRD) analysis was carried out on a Bruker D8 Advance X-ray diffractometer with $\mathrm{Cu} \mathrm{K}$ a radiation. Absorption measurements were performed with a UV-vis-NIR spectrophotometer (Perkin-Elmer Lambda 750).

\section{Ensemble absorbance:}

Simulation of the optical absorption spectrum of Te nanomaterials using Gans theory ${ }^{4}$ through the well known equations:

$$
\gamma=\frac{2 \cdot \pi \cdot \mathrm{N} \cdot \mathrm{V} \cdot \varepsilon_{\mathrm{m}}{ }^{3 / 2}}{3 \cdot \lambda} \cdot \Sigma_{\mathrm{j}} \frac{\left(1 / \mathrm{P}_{\mathrm{j}}{ }^{2}\right) \cdot \varepsilon_{2}}{\left(\varepsilon_{1}+\frac{1-\mathrm{P}_{\mathrm{j}}^{2}}{\mathrm{P}_{\mathrm{j}}} \varepsilon_{\mathrm{m}}\right)^{2}+\varepsilon_{2}{ }^{2}}
$$

$\gamma$ the extinction coefficient, $\quad N$ the number of particles per unit volume,

$V$ the particle volume,

$\varepsilon_{\mathrm{m}}$ the dielectric constant of the surrounding medium,

$\lambda$ the wavelength of the interacting light.

$\varepsilon_{1}, \quad \varepsilon_{2}$ the real and complex parts of the material dielectric function.

$P_{j}$ the depolarization factors for the three axes $\mathrm{A}, \mathrm{B}$, and $\mathrm{C}$ of the $\operatorname{rod}$ with $\mathrm{A}>\mathrm{B}=\mathrm{C}$.

Based on the previous study ${ }^{4}$, the derived relationship between the absorption $\lambda_{\max }$ 
( the maximum absorption peak ), $\mathrm{R}$ and $\varepsilon_{\mathrm{m}}$, might discribe as follow:

$$
\lambda_{\text {max }}=\eta \cdot(a \cdot \mathrm{R}+b) \cdot \varepsilon_{\mathrm{m}}+c
$$

$\eta$ normalized variable,

$a, b, c$ relative constants.

\section{Density functional theory (DFT) calculations:}

Cluster models are selected in present work, as shown in Fig S1. To avoid the influence of neighboring clusters, the crystal models used in VASP calculations should be large. As shown in Table S1, the lattice constants for each model are listed. And thus, the distances between any two clusters are more than $10 \AA$.

Table S1. The lattice constants for each model.

\begin{tabular}{|l|l|l|l|}
\hline & $\mathrm{a}$ & $\mathrm{b}$ & $\mathrm{c}$ \\
\hline $\mathrm{O}_{2}$ & 30 & 15 & 15 \\
\hline $\mathrm{CTAB}$ & 30 & 15 & 15 \\
\hline $\mathrm{Te}(1011)$ & 30 & 30 & 30 \\
\hline $\mathrm{OC}$ & 30 & 15 & 15 \\
\hline OT & 30 & 30 & 30 \\
\hline OCT & 30 & 30 & 30 \\
\hline
\end{tabular}

a

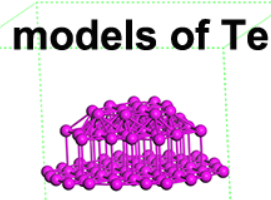

C

models of CTAB

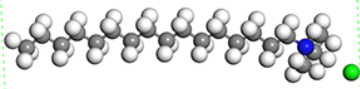

e



b models of $\mathrm{O}_{2}$

d models of $\mathrm{Te}+\mathrm{O}_{2}$

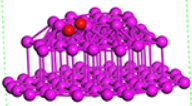

f models of OTC

a

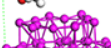

Figure S1 Cluster models in present work. 

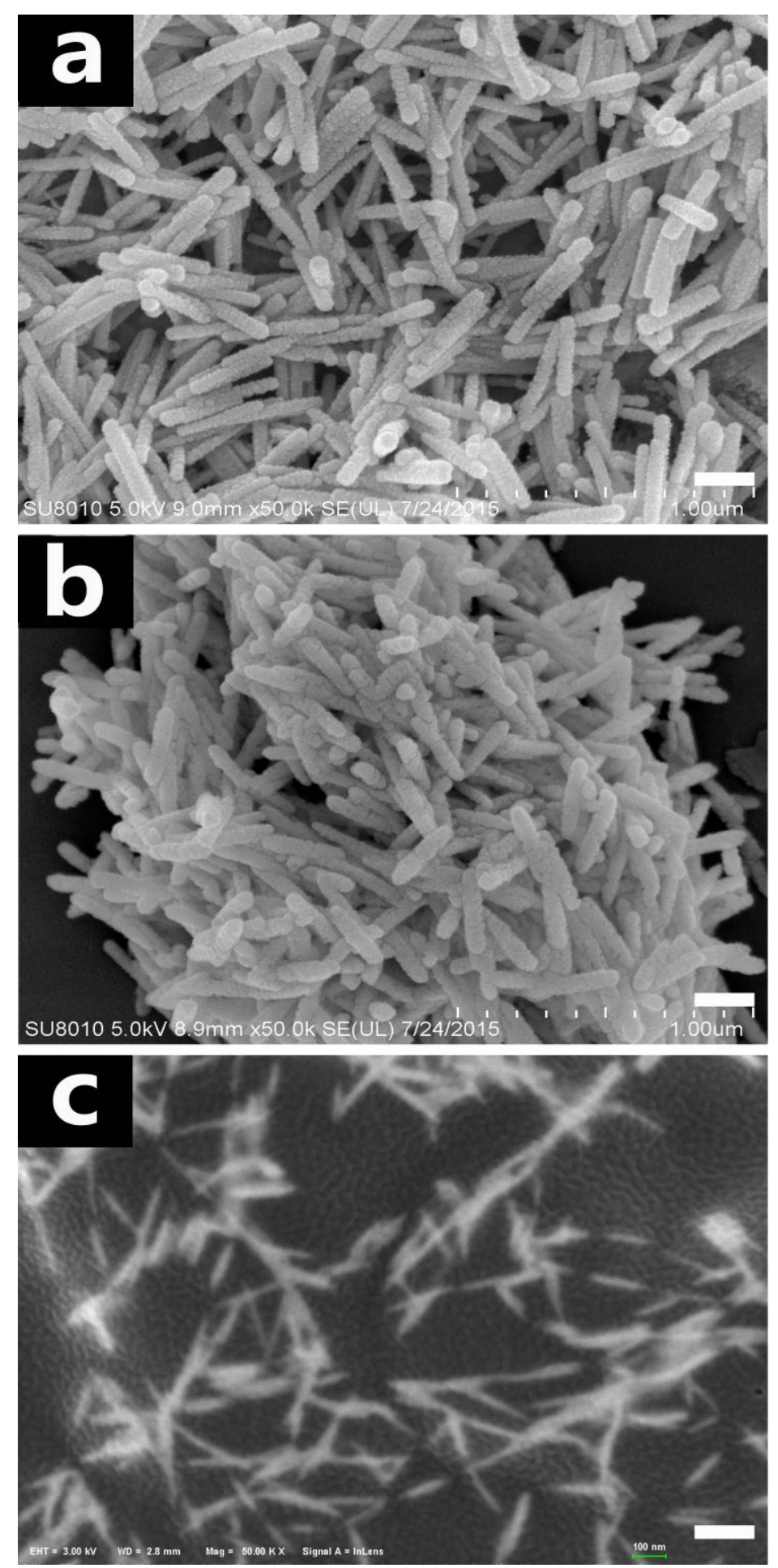

Figure S2 FESEM images of a) TeNRs, b) Te nano-intermediate substance, c) TeNFs. Scale bar: $100 \mathrm{~nm}$. 


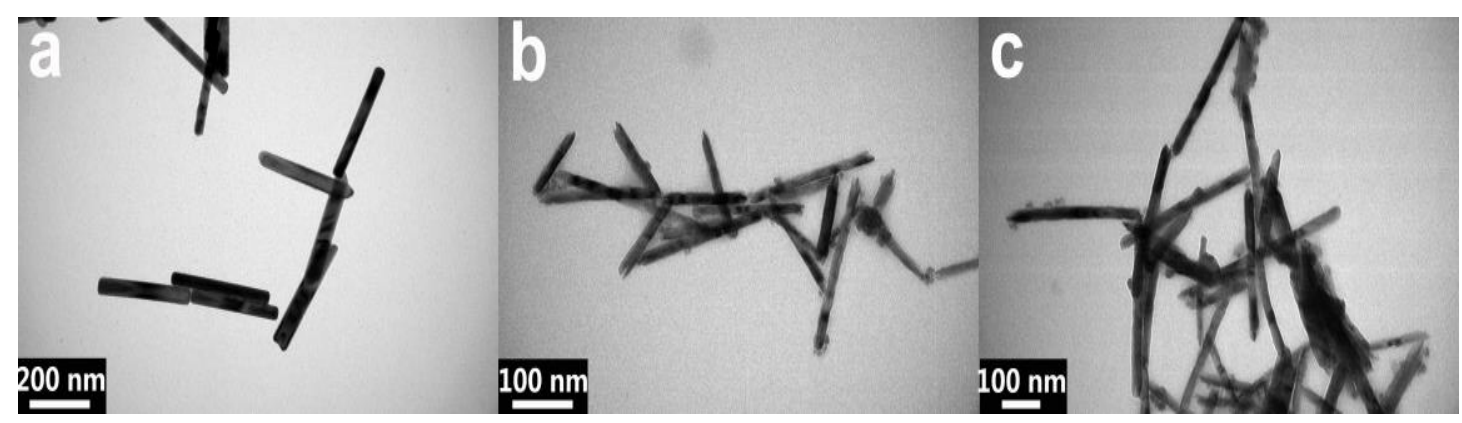

Figure $\mathbf{S 3}$ a) TEM images of TeNRs. TEM images of the structure of TeNRs under b) oxygen and c) nitrogen-saturated conditions at $60^{\circ} \mathrm{C}$ with constant magnetic stirring.



Figure S4 TEM images of Te nano-architectures before a) and after b) dispersed in 10 $\mathrm{mL}$ SDS solution at $60^{\circ} \mathrm{C}$ for 110 min under constant magnetic stirring. 


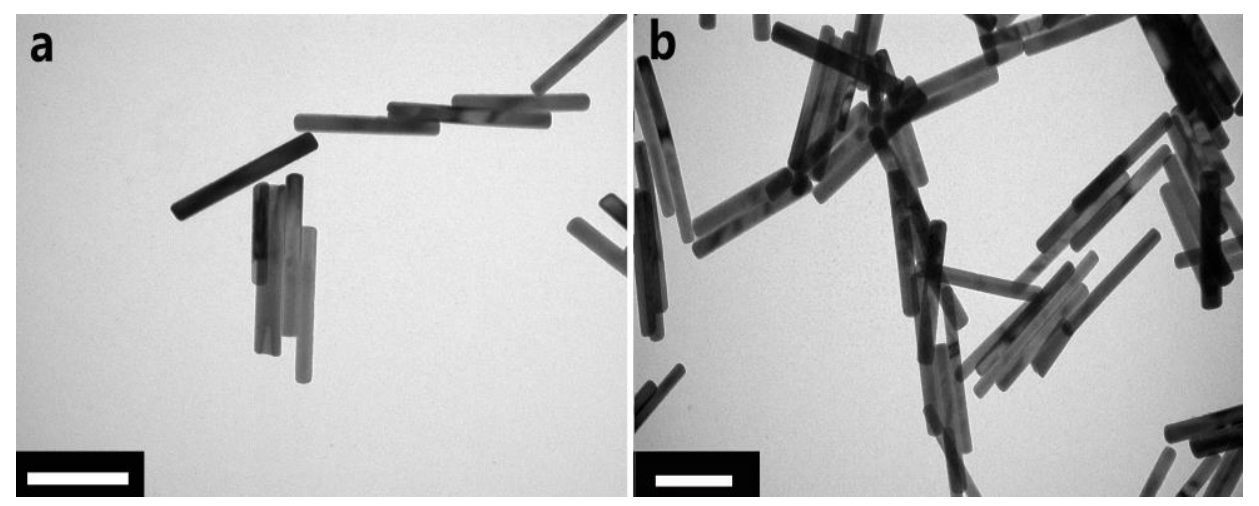

Figure S5 a) TEM images of TeNRs. b) TEM images of TeNRs after $110 \mathrm{~min}$ dispersed in $10 \mathrm{~mL} \mathrm{H}_{2} \mathrm{O}$ at $60^{\circ} \mathrm{C}$ under constant magnetic stirring. Scale bar: $200 \mathrm{~nm}$.

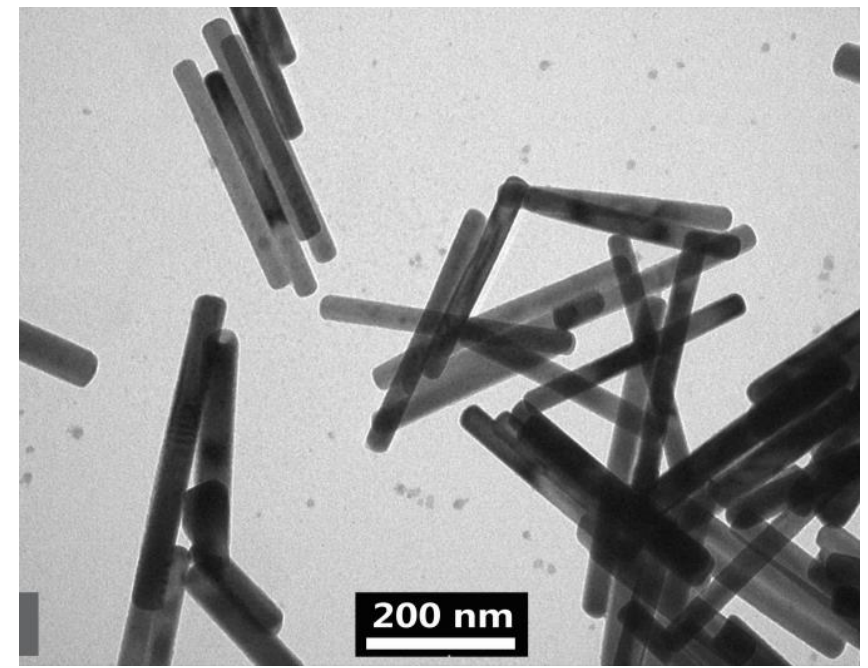

Figure S6 TEM image of TeNRs. 



d

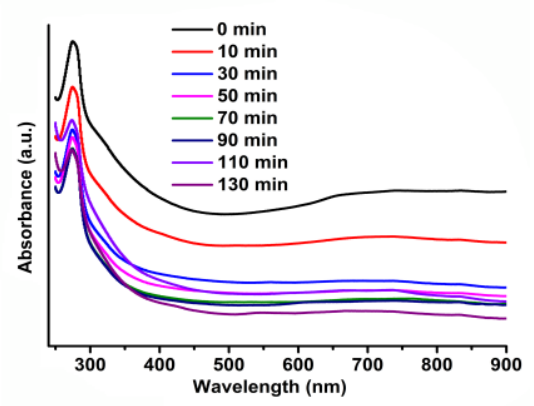

Figure S7 Changes in absorption spectra of substrate with catalytic time of $0 \mathrm{~min}$, $10 \mathrm{~min}, 30 \mathrm{~min}, 50 \mathrm{~min}, 70 \mathrm{~min}, 90 \mathrm{~min}, 110 \mathrm{~min}, 130 \mathrm{~min}$ (from top to bottom) under different CTAB concentrations a) $0 \mathrm{mM}$, b) $13.7 \mathrm{mM}$, c) $41.2 \mathrm{mM}$, d) $54.9 \mathrm{mM}$. 

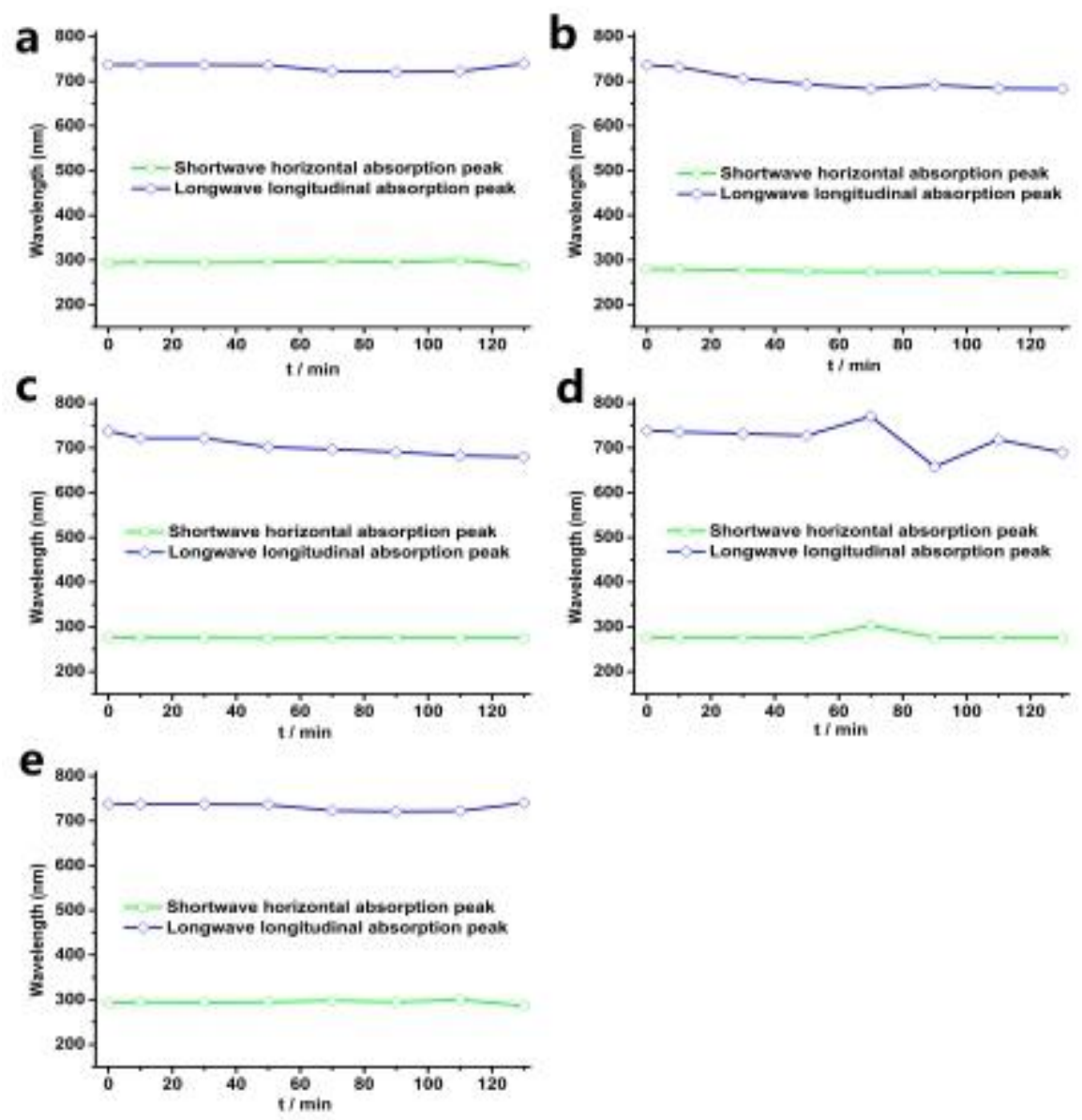

Figure S8 The change of longitudinal absorption peak position $\left(\lambda_{\max }\right)$ and lateral absorption peak $\left(\lambda_{\min }\right)$ with the time of $0 \mathrm{~min}, 10 \mathrm{~min}, 30 \mathrm{~min}, 50 \mathrm{~min}, 70 \mathrm{~min}, 90$ min, $110 \mathrm{~min}, 130 \mathrm{~min}$ under different CTAB concentrations a) $0 \mathrm{mM}$, b) $13.7 \mathrm{mM}$, c) $27.5 \mathrm{mM}$, d) $41.2 \mathrm{mM}$, e) $54.9 \mathrm{mM}$. 
Table S2. The calculated data of the total energy for the several representative states.

\begin{tabular}{|l|l|}
\hline Structures & Energy $(\mathrm{eV})$ \\
\hline $\mathrm{O}_{2}$ & -8.724 \\
\hline $\mathrm{CTAB}$ & -337.910 \\
\hline $\mathrm{Te}(1011)$ & -258.813 \\
\hline OC & -347.025 \\
\hline OT & -258.848 \\
\hline OCT & -606.992 \\
\hline
\end{tabular}

